\title{
ИЗОТЕРМА СОРБЦИИ ГИДРОХЛОРИДА ПИРИДОКСИНА НА ГЛАУКОНИТЕ
}

\author{
(C) 2017 Д. Л. Котова, Т. А. Крысанова, М. С. Василенко, М. Н. Артамонова, С. Ю. Васильева \\ Воронежский государственный университет, Университетская пл., 1, 394018 Воронеж, Россия \\ e-mail:takrys@yandex.ru
}

Поступила в редакцию 12.04.2017 г.

\begin{abstract}
Аннотация. Выявлены и объяснены закономерности сорбции гидрохлорида пиридоксина на глауконите. Изотермы сорбции проанализированы с применением теорий Ленгмюра, Фрейндлиха, Редлиха-Петерсона и Брунауэра-Эммета-Теллера (БЭТ). Установлено, что процесс сорбции включает в себя локализацию катиона пиридоксина у отрицательно заряженных центров глауконита в результате ионного обмена с внекаркасными катионами и образование ассоциатов. Сорбция пиридоксина по механизму ионного обмена с максимальной вероятностью описывает модель Ленгмюра.
\end{abstract}

Ключевые слова: сорбция, глауконит, гидрохлорид пиридоксина, ионный обмен.

\section{ВВЕДЕНИЕ}

Нанопористые природные алюмосиликаты являются перспективными сорбентами для решения прикладных задач, таких как разделение, выделение и концентрирование биологически активных соединений (БАВ), а также количественного определения их в биологических средах [1-6]. Одним из представителей алюмосиликатов является глауконит. Интерес к применению глауконита в качестве сорбента обусловлен его структурой, развитой поверхностью и высокой сорбционной емкостью [7-10]. Отмечается, что специфичность сорбция БАВ, в частности витаминов, на алюмосиликатах определяется энергиями взаимодействиями различных типов, структурой и химическими свойствами сорбента и сорбата. Гидрохлорид пиридоксина (витамин $\mathrm{B}_{6}$ ) является важным биологически активным соединением, необходимым для нормальной жизнедеятельности организма [11]. Данная работа посвящена исследованию закономерности сорбции гидрохлорида пиридоксина на глауконите.

\section{МЕТОДИКА ЭКСПЕРИМЕНТА}

В качестве объекта исследования выбран глинистый алюмосиликат - глауконит со слоистой структурой месторождения юго-запада Воронежской антеклизы. Фазовый состав глауконита представлен смешаннослойными минералами ряда иллит-смектит с соотношением слоев 20:80 и высоким содержанием железа. В межслоевом про- странстве размещены молекулы воды и обменные катионы: $\mathrm{K}^{+}, \mathrm{Mg}^{2+}$ и $\mathrm{Ca}^{2+}$, которые компенсируют отрицательный заряд матрицы сорбента. Суммарный объем пор $-0.31 \mathrm{~cm}^{3} /$, средний диаметр пор 5.7 нм, удельная поверхность, определенная по адсорбции молекул воды - $126.8 \mathrm{~m}^{2} / \Gamma[12,13]$.

В работе использовали гидрохлорид пиридоксина (PN) (2-метил-3-окси-4,5-ди-(оксиметил)пиридина гидрохлорид) производства AppliChem, чистота $99.8 \%$. Структурная формула приведена на рис. 1.<smiles>Cc1ncc(CO)c(CO)c1O</smiles>

Рис. 1. Структурная формула гидрохлорида пиридоксина

[Fig. 1. The structural formula of pyridoxine hydrochloride]

Экспериментальное исследование равновесия в системе глауконит (фракция 0.02-0.06 мм) - водный раствор гидрохлорида пиридоксина проводили при температуре $295 \pm 2$ К в статических условиях методом переменных концентраций. Интервал используемых концентраций витамина составил 0.020-7.250 ммоль/дм ${ }^{3}$. В исследуемой области концентраций (значение рН раствора 3.52 
3.94) гидрохлорид пиридоксина присутствует в растворе преимущественно в виде катиона [14]. Предварительно определив влажность, глауконит массой $0.10 \pm 0.0002$ г в воздушно-сухом состоянии приводили в контакт с $200.0 \mathrm{~cm}^{3}$ водного раствора витамина известной концентрации и выдерживали до установления равновесия в системе при заданной температуре и постоянном перемешивании. Время установления равновесия в исследуемой системе, определенное из предварительного кинетического эксперимента, составило 60 минут. Равновесные фазы разделяли фильтрованием. Равновесный раствор анализировали на содержание витамина $\mathrm{B}_{6}$ спектрофотометрическим методом на спектрофотометре СФ-16 при длине волны $\lambda_{\text {РN }}=292$ нм ( $\varepsilon=6504$ дм $^{3} /($ моль $е$ м $), S_{r}=$ $0.003)$, которая отвечает присутствию в водном растворе катиона пиридоксина. Количество сорбированного витамина устанавливали по разности концентраций раствора до и после контакта с сорбентом. В равновесном растворе определяли содержание участвующих в ионном обмене внекаркасных катионов: $\mathrm{K}^{+}$(методом пламенной фотометрии, ошибка 2.2 \%), $\mathrm{Ca}^{2+}$ и $\mathrm{Mg}^{2+}$ (методом комплексонометрии, ошибка 0.3 \%). Экспериментальные результаты обработаны методом математической статистики при доверительной вероятности 0.95 .

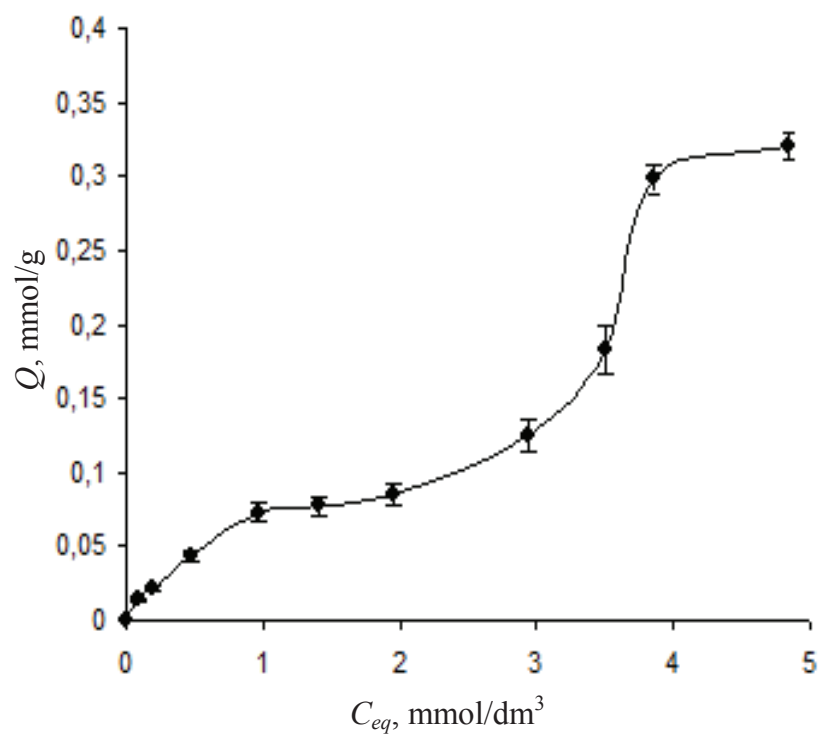

Рис. 2. Изотерма сорбции гидрохлорида пиридоксина на глауконите от концентрации равновесного раствора при $295 \mathrm{~K}$

[Fig. 2. The isotherm of sorption of pyridoxine hydrochloride on glauconite from the concentration of the equilibrium solution at $295 \mathrm{~K}$ ]
ИК-спектры гидрохлорида пиридоксина, глауконита до и после контакта с водным раствором сорбата регистрировали на спектрометре Equinox 55 (Bruker) с Фурье-преобразованием в режиме диффузионного отражения в интервале час-

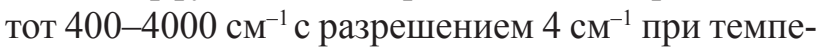
ратуре 298 К. Ошибка метода 1-3 \%. Интерпретацию полученных ИК-спектров осуществляли, используя литературу [14-16].

\section{ОБСУЖДЕНИЕ РЕЗУЛЬТАТОВ}

Межфазное равновесное распределение гидрохлорида пиридоксина в сорбционном процессе оценивали на основе изотермы сорбции (рис. 2).

По классификации Джайлса изотерма соответствует S-форме [17], что указывает на изменение в природе межмолекулярных взаимодействий в исследуемой системе. Механизм сорбции пиридоксина обуславливает состояние молекул в водном растворе (катионная, нейтральная или анионная форма) [8]. В области концентраций раствора $<2.0$ ммоль/дм ${ }^{3}$ сорбция катиона пиридоксина протекает с выделением в равновесный раствор эквивалентного количества обменных катионов $\left(\mathrm{K}^{+}\right.$, $\left.\mathrm{Ca}^{2+}, \mathrm{Mg}^{2+}\right)$ (рис. 3).

Большее участие в ионном обмене отмечается для иона калия, который является основным внекаркасным катионом в структуре глауконита [13].

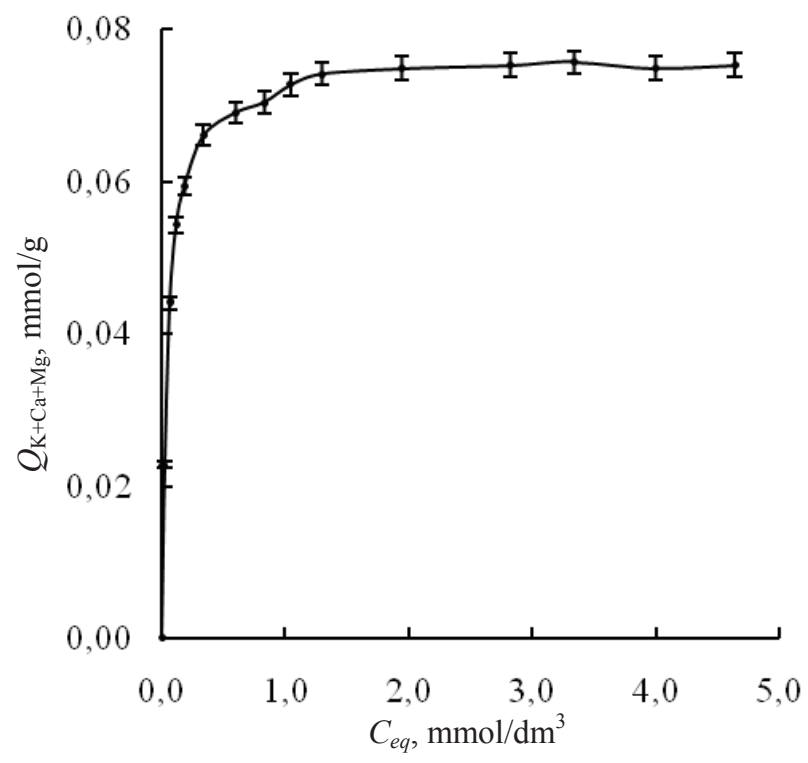

Рис. 3. Зависимость обменной составляющей сорбции катиона пиридоксина на глауконите

[Fig. 3. Dependence of the exchange component sorption on cation pyridoxine glauconite] 
Количество закрепленного на сорбенте витамина по механизму ионного обмена возрастает пропорционально его концентрации в растворе, а затем изменяется незначительно, на кривой наблюдается плато. Адсорбция является локализованной. Формирование монослоя пиридоксина происходит на электроотрицательных центрах алюмокислородного каркаса сорбента. Значение обменной емкости составляет 0.080 ммоль/г (1.44 мг/г), что примерно в 4 раза меньше сорбционной емкости монослоя по катиону пиридоксина для клиноптилолита (0.30 ммоль/г) [18]. Это может быть обусловлено размерным фактором, а именно недоступностью части заряженных центров в структуре сорбента для пиридоксина.

С ростом концентрации раствора пиридоксина $\left(C>2.5\right.$ ммоль/дм $\left.{ }^{3}\right)$ наблюдается резкое увеличение сорбционного параметра, при этом ионообменная составляющая сорбции не изменяется. Формирование полимолекулярных слоев сорбата начинается с мономолекулярного заполнения, а дальнейший их рост в результате образования ассоциатов носит кооперативный характер. Максимальная сорбционная емкость глауконита по пиридоксину составляет 0.32 ммоль/Г (54.1 мг/г), что в полтора раза меньше, чем при сорбции на клиноптилолите (0.53 ммоль/г) и достигается из раствора меньшей концентрации [18]. Можно предположить, что образование ассоциатов пиридоксина в поровом пространстве сорбента определяют ионообменный и размерный факторы $[12,13]$.

Присутствие пиридоксина в структуре глауконита проявляется на ИК спектре появлением максимумов при 1460 и $1441 \mathrm{~cm}^{-1}$, отвечающих валентным колебаниям пиридинового кольца сорбата. Валентные колебания $\mathrm{OH}-\left(\mathrm{CH}_{2}-\mathrm{OH}\right)$ и
$\mathrm{N}^{+}$-Н-групп витамина отмечаются соответственно при 3203 и $2795 \mathrm{~cm}^{-1}$. Максимум поглощения при $1512 \mathrm{~cm}^{-1}$ соответствует деформационным колебаниям $\mathrm{N}^{+}$-Н-группы. Деформационные колебания фенольной гидроксильной группы характеризуются наличием полос поглощения при 1398 и $1210 \mathrm{~cm}^{-1}$ [15]. Монослойное закрепление катионов пиродоксина на отрицательно заряженных центрах глауконита в результате электростатического взаимодействия с положительно заряженными $\mathrm{N}^{+}$-Н-группами сорбата на ИК спектре отражается в низкочастотном сдвиге полос поглощения, относящихся к валентным колебаниям групп Si-O-Al глауконита $\left(1107 \rightarrow 1039 \mathrm{~cm}^{-1}\right)$ и $\mathrm{N}^{+}-\mathrm{H}-г$ гупп пиридоксина $\left(1543 \rightarrow 1512 \mathrm{~cm}^{-1}\right)$. Структура образующихся ассоциатов пиридоксина определяется образованием водородной связи [19], что отмечается появлением максимумов поглощения при 1398 и $1204 \mathrm{~cm}^{-1}$, отвечающих О...Н связи, и смещением полосы поглощения, характеризующей О-Н-группу в низкочастотную область спектра $\left(3222 \rightarrow 3200 \mathrm{~cm}^{-1}\right)$. Ассоциация пиридоксина в результате перекрывания $\pi$-электронной плотности пиридинового кольца [20] находит отражение в смещении его максимума поглощения в область более низких значений $\left(1471 \rightarrow 1460 \mathrm{~cm}^{-1}\right)$.

Для количественного описания равновесия сорбции в интервале концентраций, отвечающих образованию монослоя витамина, были использованы линеаризованные уравнения моделей Ленгмюра, Фрейндлиха, Редлиха-Петерсона, а для описания всей изотермы применяли теорию БЭТ (табл. 1) [21-24].

О применимости моделей судили по значению среднеквадратичного отклонения $\left(R^{2}\right)$. Линейное уравнение Редлиха-Петерсона не может быть при-

Таблица 1. Модели, применяемые для описания изотермы сорбции гидрохлорида пиридоксина

[Table 1. Models used to describe the sorption isotherm of pyridoxine hydrochloride]

\begin{tabular}{|c|c|c|}
\hline $\begin{array}{l}\text { Модель } \\
\text { [model] }\end{array}$ & $\begin{array}{c}\text { Уравнение } \\
\text { [the equation] }\end{array}$ & $\begin{array}{c}\text { Линейная форма } \\
\text { [linear form] }\end{array}$ \\
\hline Ленгмюра [Langmuir] & $Q=\frac{Q_{\infty} K_{L} C_{p}}{1+K_{L} C_{p}}$ & $\frac{1}{Q}=\frac{1}{Q_{\infty} K_{L} C_{p}}+\frac{1}{Q_{\infty}}$ \\
\hline Фрейндлиха [Freundlich] & $Q=K_{F} C_{P}^{1 / n}$ & $\ln Q=\ln K_{F}+\frac{1}{n} \ln C_{p}$ \\
\hline $\begin{array}{c}\text { Редлиха-Петерсона [Redlich- } \\
\text { Peterson] }\end{array}$ & $Q=\frac{K_{R} C_{p}}{1-a_{R} C_{p}^{\beta}}$ & $\ln \left(K_{R} \frac{C_{p}}{Q}-1\right)=\ln a_{R}+\beta \ln C_{p}$ \\
\hline $\begin{array}{c}\text { БЭТ } \\
\text { [Brunauer-Emmett-Teller] }\end{array}$ & $Q=\frac{Q_{\infty} K_{L} C_{p}}{\left(1-K_{S} C_{p}\right)\left(1-K_{S} C_{p}+K_{L} C_{p}\right)}$ & $\frac{C_{p}}{Q\left(1-K_{S} C_{p}\right)}=\left(\frac{K_{L}-K_{S}}{Q_{\infty} K_{L}}\right) C_{p}+\frac{1}{Q_{\infty} K_{L}}$ \\
\hline
\end{tabular}


менено для определения констант, так как оно содержит три неизвестных параметра, поэтому использовали процедуру минимизации (OrignPro 7.5) [25].

В табл. $1 Q$ - количество сорбируемого гидрохлорида пиридоксина, ммоль/г; $Q_{\infty}-$ предельное количество сорбированного катиона пиридоксина (емкость монослоя), ммоль/г; $C_{\mathrm{p}}$ - равновесная концентрация раствора, ммоль/дм ${ }^{3} ; K_{L}-$ константа сорбционного равновесия, характеризующая интенсивность процесса сорбции, дм³/ммоль; $n-$ константа изотермы Фрейндлиха, показывающая интенсивность сорбции; $K_{\mathrm{F}}$ - константа изотермы Фрейндлиха, соответствующая сорбционной емкости $\left(\text { дм }^{3}\right)^{1 / n}$ (ммоль $)^{1-1 / n} / \Gamma ; a_{\mathrm{R}}$ и $K_{\mathrm{R}}$ - константы изотермы Редлиха-Петерсона, $\left(\text { дм }^{3}\right)^{\beta} /$ ммоль $^{\beta}$ и дм ${ }^{3} / \Gamma$ соответственно; $\beta$ - константа, значение которой должно лежать в интервале $0<\beta<1$; $K_{\mathrm{S}}-$ константа сорбционного равновесия при образовании полимолекулярного слоя, дм³

Значения коэффициентов корреляции при линеаризации изотермы показывают, что процесс ионного обмена катиона пиридоксина с внекаркасными катионами с максимальной вероятностью описывает уравнение Лэнгмюра (табл. 2).

Применимость модели в данном случае указывает на равноценность активных центров и однородность поверхности глауконита. Значение параметра $\beta$ в уравнении Редлиха-Петерсона близкое к 1 , является подтверждением, что сорбция витамина из разбавленных растворов протекает по механизму Лэнгмюра. Анализ изотермы с использова- нием модели БЭТ позволил установить, что уравнение в линейном виде удовлетворительно описывает процесс сорбции катиона пиродоксина на глауконите в интервале концентраций 1.00-3.50 ммоль/дм³ а при дальнейшем возрастании концентрации раствора наблюдается отклонение от линейности. Величины предельной емкости монослоя $\left(Q_{\infty}\right)$ и значения $K_{L}$ для гидрохлорида пиридоксина, рассчитанные с помощью моделей Ленгмюра и БЭТ, сопоставимы.

Рассчитан равновесный коэффициент распределения $(D)$ пиридоксина в структуре глауконита. Резкое снижение его значения с ростом концентрации раствора отмечается в области формирования монослоя, что связано с влиянием стерического фактора. Полимолекулярная сорбция витамина характеризуется незначительным изменением коэффициента распределения (рис. 4).

\section{ЗАКЛЮЧЕНИЕ}

Исследованы закономерности сорбции гидрохлорида пиридоксина из водного раствора на глауконите. Для описания процесса были использованы уравнения Лэнгмюра, Фрейндлиха, РедлихаПетерсона и БЭТ. В области разбавленных растворов витамина (1.0-2.0 ммоль/дм³ ) изотерма с максимальной вероятностью описывается моделью Лэнгмюра. Показано, что формирование мономолекулярного слоя пиридоксина на глауконите протекает в результате ионного обмена катионов пиридоксина с внекаркасными катионами сорбента. Полимолекулярный характер сорбции с ростом

Таблица 2. Значения сорбционных параметров, рассчитанные с использованием уравнений Ленгмюра, Фрейндлиха, Редлиха-Петерсона и БЭТ

[Table 2. The values of the sorption parameters calculated using the equations of Langmuir, Freundlich, RedlichPeterson, and Brunauer-Emmett-Teller (BET)]

\begin{tabular}{|c|c|c|c|c|}
\hline \multicolumn{5}{|c|}{ Модель Ленгмюра [Langmuir model] } \\
\hline \multirow{2}{*}{$\begin{array}{l}\text { Гидрохлорид пиридоксина } \\
\text { [pyridoxine hydrochloride] }\end{array}$} & \multicolumn{2}{|c|}{$K_{L}$, дм ${ }^{3} /$ моль } & $Q_{\infty}$, ммоль/Г & $R^{2}$ \\
\hline & \multicolumn{2}{|c|}{2.21} & 0.08 & 0.99 \\
\hline \multicolumn{5}{|c|}{ Модель Фрейндлиха [Freundlich model] } \\
\hline \multirow{2}{*}{ Гидрохлорид пиридоксина } & \multicolumn{2}{|c|}{$K_{F},\left(\text { дм}^{3}\right)^{1 / n}(\text { ммоль })^{1-1 / n} / \Gamma$} & $N$ & $R^{2}$ \\
\hline & \multicolumn{2}{|c|}{2.51} & 3.00 & 0.92 \\
\hline \multicolumn{5}{|c|}{ Модель Редлиха-Петерсона [Redlich-Peterson model] } \\
\hline \multirow{2}{*}{ Гидрохлорид пиридоксина } & $K_{R}$, дм $^{3} / \Gamma$ & \multicolumn{2}{|c|}{$a_{R^{\prime}}\left(\text { дм }^{3}\right)^{\beta} /$ ммоль $^{\beta}$} & $R^{2}$ \\
\hline & 1.25 & \multicolumn{2}{|c|}{2.52} & 0.96 \\
\hline \multicolumn{5}{|c|}{ Модель БЭТ [Brunauer-Emmett-Teller (BET) model] } \\
\hline \multirow{2}{*}{ Гидрохлорид пиридоксина } & 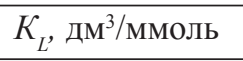 & \multicolumn{2}{|c|}{$Q_{\infty}$, ммоль $/ \Gamma$} & $R^{2}$ \\
\hline & 2.24 & \multicolumn{2}{|c|}{0.09} & 0.98 \\
\hline
\end{tabular}




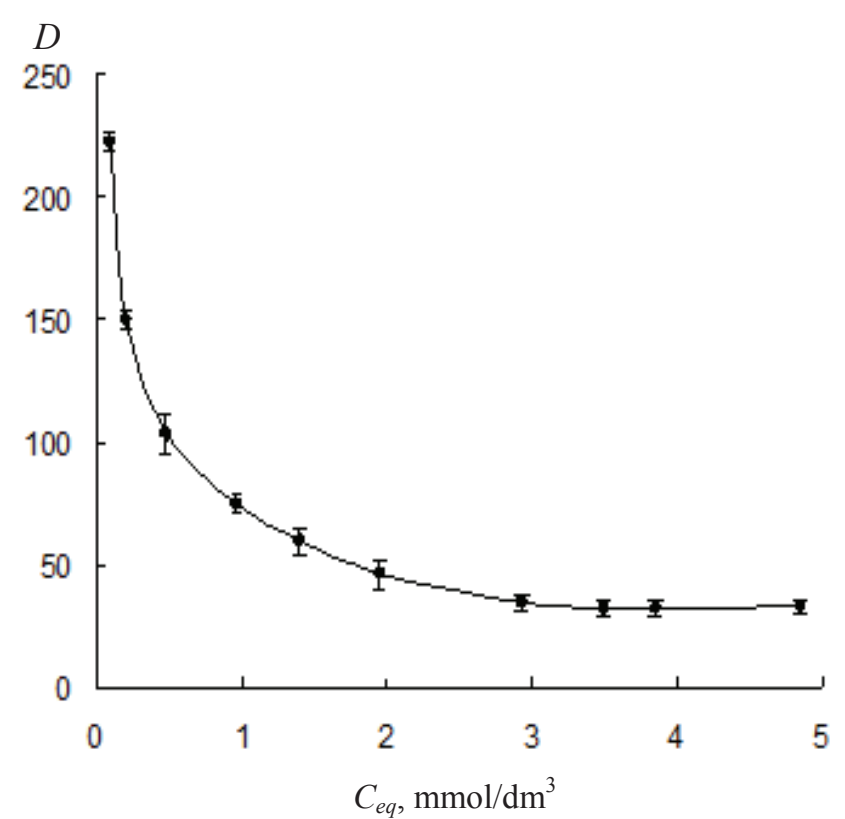

Рис. 4. Зависимость коэффициента распределения пиридоксина гидрохлорида от равновесной концентрации витамина

[Fig. 4. Dependence of the distribution coefficient $(D)$ of pyridoxine hydrochloride on the equilibrium concentration of vitamin]

концентрации раствора определяет формирование ассоциатов пиридоксина в результате образования водородной связи и $\pi-\pi$-взаимодействий пиридинового кольца.

\section{СПИСОК ЛИТЕРАТУРЫ}

1. Rivera A., Farias T. P. // Micropor. Mesopor. Mater., 2005, vol. 80, p. 337.

2. Krohn J. E., Tsapatsis M. // Langmuir, 2006, vol. 22, p. 9350.

3. Rejinojd N. S., Muthunarayanan M., Muthuchelian K., Chennazhi K. P., Nair S. V., Jayakumar R. // Carbohyd. Polym., 2011, vol. 84, p.407.

4. Basic Z., Kilibarda V., Dobri S., Resanovi R. // Vojnosanitetski Pregled, 2011, vol. 68, p.15.

5. Patel H. A., Somani R. S., Bajaj H. C., Jastra R. V. // Bull. of Mater. Sci., 2006, vol. 29, p. 133.

6. Joshi G. V., Kevadiya B. D., Patel H. A., Bajaj H. C., Jastra R. V. // Inter. J Pharm., 2009, vol. 374, p. 53.
7. Тарасевич Ю. И, Овчаренко Ф. Д. Адсорбция на глинистых минералах. Киев, Наукова думка, 1975, $275 \mathrm{p}$.

8. Ristila M., Matxain J. M., Strid A., Eriksson L. A. // J. Phys. Chem. B, 2006, vol. 110, p. 16774.

9. Котова Д. Л., До Тхи Лонг, Крысанова Т. А., Селеменев В. Ф. // Журн. физ. химии, 2011, т. 85, № 12, c. 2365 .

10. Suriyanon N., Punyapalakul P., Ngamcharussrivichai C. // Chem. Eng. J., 2013, vol. 214, p. 208.

11. Морозов Ю. В., Бажулина Н. П. Электронное строение, спектроскопия и реакиионная способность молекул. Нуклеиновые основания, витамины $B_{6} u$ их аналоги. М.: Наука, 1989, 288 с.

12. Kotova D. L., Artamonova M. N., Krysanova T. A, Novikova L. A, Belchinskaya L. I. // Сорбцчионные и хроматографические прочессы, 2016, т. 16, вып. 3, с. 390.

13. Novikova L. A., Belchinskay L. I., Krupskaya V., Roessntr F., Zhabin A. V. // Сорбциинные и хроматографические прочессы, 2015, т. 15, вып. 5, с. 730.

14. Наканиси К. Инфракрасная спектроскопия $u$ строение органических соединений. М.: Мир, 1987. $220 \mathrm{c}$.

15. Cinta S., Morari C., Vogel E., Mania D. // Vibrational Spectroscopy, 1999, vol. 19, p. 329.

16. Киселев А. В. Инфракрасные спектры поверхностных соединений и адсорбиионных вещчеств. М.: Наука, 1972. 459 с.

17. Sing K. S. W., Everett D. H., Haul R. A.W., et al. // Pure and Applied Chemistry, 1985, vol. 57, № 4, p. 603.

18. Котова Д. Л., Фам Тхи Гам, Крысанова Т. А., Васильева С. Ю. // Коллоид. журнал, 2016, т. 78, № 1, c. 54 .

19. Hanic F. // Acta Cryst., 1996, vol. 21, p. 332.

20. Пюльман Б. Межмолекулярные взаимодействия: от двухатомных молекул до биополимеров. М.: Мир, $1981.592 \mathrm{c}$.

21. Langmuir I. // J. Am. Chem. Soc., 1916, vol. 38, p. 2221.

22. Freundlich H. // J. Phys. Chem., 1906, vol. A 57, p. 385 .

23. Redlich O., Peterson D. L. // J. Phys. Chem., 1959, vol. 63, p. 1024.

24. Amanollah E., Jafar S., Soltan M. // Adsorption, 2009, vol. 15, № 1, p. 65 .

25. Allen S. J., Gan Q., Matthews R., Johnson P.A. // Bioresource Technology, 2003, vol. 88, № 2, p. 143. 


\title{
SORPTION ISOTHERM OF PYRIDOXINE HYDROCHLORIDE ON GLAUCONITE
}

\author{
(C) 2017 D. L. Kotova, T. A. Krysanova, M. S. Vasilenko, N. M. Artamonova, S. Yu. Vasilyeva \\ Voronezh State University, 1 Universitetskaya sq., 394018 Voronezh, Russia \\ e-mail:takrys@yandex.ru
}

Received 12.04.2017

\begin{abstract}
Natural aluminosilicates are promising sorbents that can be used to separate and isolate biologically active substances, namely vitamins. Hydrochloride pyridoxine (vitamin B6) is an important biologically active compound necessary for the normal functioning of the body. This article describes the investigation of absorption regularities of pyridoxine hydrochloride on the glauconite. Clay aluminosilicate, i.e. glauconite with a layered structure (from south-west of the Voronezh anteclise) was chosen as the subject for the study. Its phase composition is represented by mixed-layer minerals of illite-smectite with 20:80 ratio of layers and a high iron level. Its interlayer space has water molecules and $\mathrm{K}^{+}, \mathrm{Mg}^{2+}$ and $\mathrm{Ca}^{2+}$ exchangeable cations.

The study of equilibrium in glauconite $\left(0.02-0.06 \mathrm{~mm}\right.$ fraction), i.e. aqueous solution of vitamin $\mathrm{B}_{6}$, was carried out at a temperature of $295 \mathrm{~K}$ under static conditions by using variable concentrations. The interval for the vitamin concentrations within 0.02 and $7.25 \mathrm{mmol} / \mathrm{dm}^{3}$ in the $\mathrm{pH}$ solution was 3.50-3.94, where pyridoxine hydrochloride was mainly present in the solution in the form of cations.

Absorption regularities of pyridoxine hydrochloride on the glauconite were identified and explained. To conduct a quantitative description of sorption equilibrium in the interval of concentrations that lead to the formation of the vitamin monolayer the linearized equations of Langmuir, Freundlich, Redlich-Peterson models were used. Whereas the Brunauer-Emmett-Teller (BET) theory was used to describe the isotherm as a whole.

It was established that the sorption process involves the localization of pyridoxine cations at the negatively charged centres of glauconite which results from ion exchanges with extra framework cations and the formation of associates. Pyridoxine sorption caused by ion exchange can be described with high probability by the Langmuir model. The multi-molecular nature of the sorption and the increase in the solution concentration determine the formation of pyridoxine associates caused by the formation of hydrogen bonds and $\pi-\pi$ interactions in the pyridine ring.

The equilibrium coefficient for the pyridoxine distribution in the glauconite structure was calculated. The increase in concentration of the solution leads to the sharp decrease in the formation of the monolayer. The polymolecular sorption of the vitamin is characterized by a slight change in the distribution coefficient.
\end{abstract}

Keywords: sorption, glauconite, pyridoxine hydrochloride, ion exchange.

\section{REFERENCES}

1. Rivera A., Farias T. P. Micropor. Mesopor. Mater., 2005, vol. 80, p. 337. DOI: 10.1186/2052-336X-12-35

2. Krohn J. E., Tsapatsis M. Langmuir, 2006, vol. 22, p. 9350 . DOI.org/10.1021/la061743m

3. Rejinojd N. S., Muthunarayanan M., Muthuchelian K., Chennazhi K. P., Nair S. V., Jayakumar R. Carbohyd. Polym., 2011, vol. 84, p. 407. DOI.org/10.1016/j.carbpol.2010.11.056

4. Basic Z., Kilibarda V., Dobri S., Resanovi R. Vojnosanitetski Pregled, 2011, vol. 68, p. 15. DOI: 10.2298/ VSP160212081D

5. Patel H. A., Somani R. S., Bajaj H. C., Jastra R. V. Bull. of Mater. Sci., 2006, vol. 29, p. 133. http://www.ias. ac.in/article/fulltext/boms/029/02/0133-0145
6. Joshi G. V., Kevadiya B. D., Patel H. A., Bajaj H. C., Jastra R. V. Inter. J Pharm., 2009, vol. 374, p. 53. DOI: 10.1016/j.ijpharm.2009.03.004

7. Tarasevich Yu. I, Ovcharenko F. D. Adsorption on Clay Minerals. Kiyev, Naukova Dumka Publ., 1975, $275 \mathrm{p}$.

8. Ristila M., Matxain J. M., Strid A., Eriksson L. A. J. Phys. Chem. B, 2006, vol. 110, p. 16774. DOI: 10.1021/ jp062800n

9. Kotova D. L., To Thi Long, Krysanova T. A., Selemenev V. F. J. Phys. Chem., 2011, vol. 85, no. 12, p. 2365. DOI: $10.1134 / \mathrm{S} 0036024411120168$

10. Suriyanon N., Punyapalakul P., Ngamcharussrivichai C. Chem. Eng. J., 2013, vol. 214, p. 208. DOI:10.3390/ w9040250 
11. Morozov Yu. V., Bazhulina N. P. Electronic Structure, Spectroscopy and Reactivity of Molecules. Nucleic Acid Bases, Vitamins B6 and their Analogs. Moscow, Nauka Publ., 1989, 288 p. (in Russia)

12. Kotova D. L., Artamonova M. N., Krysanova T. A, Novikova L. A, Belchinskaya L. I. Sorption and Chromatographic Processes, 2016, vol. 16, is. 3, p. 390. Available at: http://www.sorpchrom.vsu.ru/articles/20160315.pdf

13. Novikova L. A., Belchinskay L. I., Krupskaya V., Roessner F., Zhabin A. V. // Sorption and Chromatographic Processes, 2015, vol. 15, is. 5, p. 730. Available at: http:// www.sorpchrom.vsu.ru/articles/20150517.pdf

14. Nakanisi K. Infrared Spectroscopy and Structure of Organic Compounds. Moscow, Mir Publ., 1987, 220 p. (in Russia)

15. Cinta S., Morari C., Vogel E., Mania D. Vibrational Spectroscopy, 1999, vol. 19, p. 329. PII: S0924-2031 99 00019-3

16. Kiselev A.V. Infrared Spectra of Surface Compounds and Adsorbed Substances. Moscow, Nauka Publ., 1972, 459 p. (in Russia)
17. Sing K. S. W., Everett D. H., Haul R. A.W., et al. Pure and Applied Chemistry, 1985, vol. 57, no. 4, p. 603. DOI: 10.12691/nnr-1-2-2

18. Kotova D. L., Fam Thi Gam, Krysanova T. A., Vasilyeva S. Yu. Colloid. Journal, 2016, vol. 78, no. 1, p. 54. DOI: $10.7868 / \mathrm{S} 0023291215060130$

19. Hanic F. Acta Cryst., 1996, vol. 21, p. 332.

20. Pyul'man B. Intermolecular Interaction: From Diatomic Molecules to Biopolymers. Moscow, Mir Publ., 1981. 592 p. (in Russia)

21. Langmuir I. J. Am. Chem. Soc., 1916, vol. 38, p. 2221.

22. Freundlich H. J. Phys. Chem., 1906, vol. A 57, p. 385 .

23. Redlich O., Peterson D.L. J. Phys. Chem., 1959, vol. 63, p. 1024.

24. Amanollah E., Jafar S., Soltan M. Adsorption, 2009, vol. 15, no. 1, p. 65. DOI: 10.1007/s10450-009-9151-3

25. Allen S. J., Gan Q., Matthews R., Johnson P. A. Bioresource Technology, 2003, vol. 88, no. 2, p. 143. DOI: 10.1016/S0960-8524(02)00281-X
Котова Диана Липатьевна - д. х. н., профессор кафедры аналитической химии, Воронежский государственный университет; тел.: +7(4732) 208932

Крысанова Татьяна А. - к. х. н., доцент кафедры аналитической химии, Воронежский государственный университет; тел.: +7(4732) 208932, e-mail: takrys@ yandex.ru

Василенко Мария С. - студентка 5 курса кафедры аналитической химии, Воронежский государственный университет; тел.: +7(4732) 208932

Артамонова Мария Н. - студентка 5 курса кафедры аналитической химии, Воронежский государственный университет; тел.: +7(4732) 208932

Васильева Светлана Ю. - к. х. н., ассистент кафедры общей и неорганической химии, Воронежский государственный университет; тел.: +7(4732) 208932
Kotova Diana L. - Dr. Sci. (Chem.), Professor of the Analytical Chemistry Department, Voronezh State University; ph.: +7(4732) 208932

Krysanova Tatiana A. - Cand. Sci. (Chem.), Lecturer of the Analytical Chemistry Department, Voronezh State University; ph.: +7(4732) 208932, e-mail: takrys@yandex. $\mathrm{ru}$

Vasilenko Maria S. - student of the Analytical Chemistry Department, Voronezh State University; ph.: +7(4732) 208932

Artamonova Maria N. - student of the Analytical Chemistry Department, Voronezh State University; ph.: +7(4732) 208932

Vasilyeva Svetlana S. Yu. - Cand. Sci. (Chem.), Assistant of the Department of General and Inorganic Chemistry, Voronezh State University; ph.: +7(4732) 208932 